\title{
The hemophagocytic syndrome in an immunocompromised patient: A diagnostic challenge
}

\author{
I Fiteni $M D^{1}$, G Perez-Lungmus $M D^{2}$, JM Grasa $M D^{3}$, C Motis $M D^{4}$
}

\section{CASE PRESENTATION}

A 23-year-old woman was admitted to hospital because of intermittent fever up to $40^{\circ} \mathrm{C}$ accompanied by significant arthralgia and myalgia for the previous seven days. She had been receiving azathioprine $100 \mathrm{mg}$ per day and prednisone $60 \mathrm{mg}$ per day for Harada's disease with frequent bouts of recurrent uveitis for the past two years. She had been complaining of diffuse muscle and joint pain, stiffness and general weakness for the previous seven months, which prevented her from walking. Steroids had been tapered during the previous several weeks for suspected iatrogenic myopathy, although there had been no improvement. She denied cough, chest pain, headache or other symptoms. She had no pets, except contact with a friend's dog, and had not travelled recently. Physical examination revealed a cushingoid woman in no distress with no adenopathy. Chest, cardiovascular, skin and abdominal exams were normal, and no hepatosplenomegaly was noted. Blood analysis revealed pancytopenia (hemoglobin $5.4 \mathrm{mmol} / \mathrm{L}$; leukocytes $2 \times 10^{9} / \mathrm{L}$ $\left[0.4 \times 10^{9} / \mathrm{L}\right.$ lymphocytes]; platelets $\left.38 \times 10^{9} / \mathrm{L}\right)$, elevated

liver enzymes (aspartate aminotransferase $561 \mathrm{U} / \mathrm{L}$; alanine aminotransferase $451 \mathrm{U} / \mathrm{L}$; lactate dehydrogenase $2300 \mathrm{U} / \mathrm{L}$ ) and an increased prothrombin time by $56 \%$. The erythrocyte sedimentation rate was $120 \mathrm{~mm} / \mathrm{h}$. Total gamma globulins were decreased $(10 \mathrm{~g} / \mathrm{L})$ with low immunoglobulin $\mathrm{G}$ $(6.53 \mathrm{~g} / \mathrm{L})$ and normal albumin $(45 \mathrm{~g} / \mathrm{L})$. Triglycerides and fibrinogen were normal. Urine analysis, other routine biochemical markers and renal function were normal, as were the assays for rheumatoid factor, total complement activity, reticulocyte count and vitamin $B_{12}$ levels. Autoimmmune studies (ie, antinuclear antibody, anti-DNA) and a Coomb's test were negative, as was serology for common endemic infectious diseases (ie, Brucella species; Cytomegalovirus; Epstein-Barr virus; hepatitis A, B and C viruses; leishmania). Blood and urine cultures for bacteria were negative. A radiograph of the chest was normal, although echocardiography revealed a mild pericardial effusion. A computed tomography scan of the chest did not reveal any abnormalities. A diagnostic procedure was performed. What is the diagnosis?
\end{abstract}

${ }^{1}$ Department of Internal Medicine, Clínica Montpellier; ${ }^{2}$ Haematology consultant, Clínica Montpellier; ${ }^{3}$ Laboratorio José María Grasa Biel, Sociedad Anónima; ${ }^{4}$ Department of Neurology, Clínica Montpellier, Zaragoza, Spain

Correspondence: Dr Isabel Fiteni, Andador Luis Puntes 4, $3^{\underline{a}}$ B, 50008 Zaragoza, Spain. Telephone 0034-9-76-590412,

e-mail ifiteni@saludalia.com 


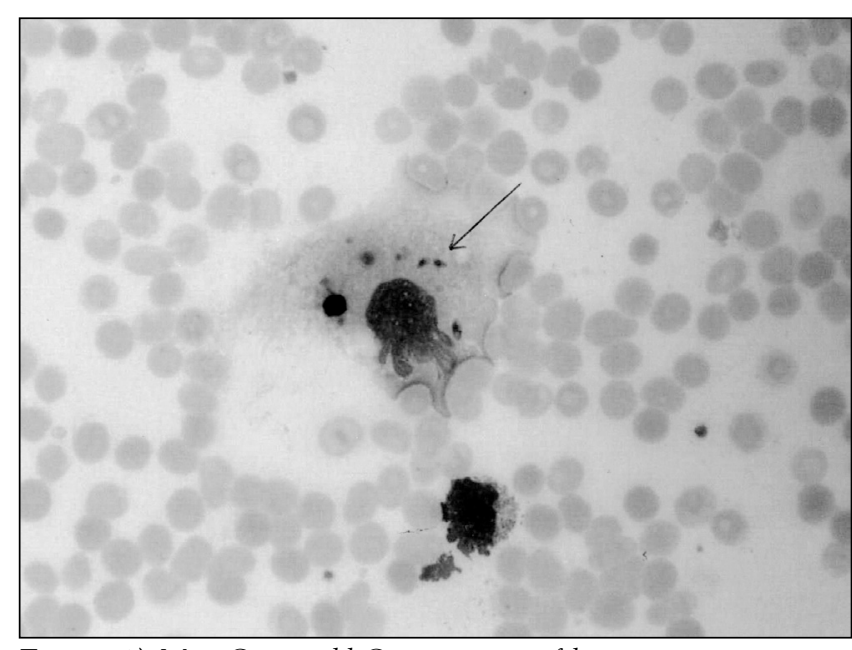

Figure 1) May-Grünwald-Giemsa stain of bone marrow aspirate, disclosing a mononuclear cell containing two Leishmania amastigotes (arrow) (Magnification $\times 1000$ )

\section{DIAGNOSIS}

A bone marrow aspirate was performed and revealed rich cellularity with evidence of profuse hemophagocytosis: focal hyperplasia of hyperreactive histiocytes containing debris of erythrocytes, myelocytes and platelets. Rare phagocytic cells showed small rounded bodies characteristic of Leishmania amastigotes (Figure 1). In fact, only two such phagocytic cells were seen after two hours of microscopic evaluation of the marrow sample. A polymerase chain reaction assay for the Mycobacterium tuberculosis complex was negative, as were cultures for both mycobacteria and Brucella species. Repeated serological tests for anti-Leishmania antibodies by means of indirect immunofluorescence remained negative during the illness. Treatment was started with meglumin antimony (Glucantime [Aventis Pharma, Spain]) $20 \mathrm{mg} / \mathrm{kg} / \mathrm{d}$, after which all symptoms subsided and the pancytopenia recovered. However, the treatment was discontinued after 13 days due to the development of drug-induced pancreatitis, and therapy was initiated with amphotericin B at a dose of $0.5 \mathrm{mg} / \mathrm{kg} / \mathrm{d}$ for six weeks, which was well tolerated. A bone marrow aspirate was repeated one month later, which was interpreted as normal, showing no hemophagocytosis nor protozoa. Anti-Leishmania serology became weakly positive (titre of $1: 40$ ) one month after discontinuing the treatment. The patient recovered completely 10 months after the onset of disease, having received a total of two months of therapy. After one year of followup, she remains well, receiving only low chronic doses of azathioprine.

\section{DISCUSSION}

Hemophagocytic syndrome is characterized by uncontrolled nonmalignant proliferation of histiocytes and macrophages in bone marrow, lymphoid tissues or other organs, accompanied by clinical events such as fever, lymphadenopathy or hepatosplenomegaly. Pancytopenia develops due to activation of the mononuclear phagocytic system, with overproduction of cytokines and phagocytosis of erythrocytes, platelets and leukocytes (1). The histopathological and cytological findings of hemophagocytosis may be found in conjunction with malignant reactive histiocytosis, hystiocytic medullary reticulosis, familial lymphohistiocytosis, or sinus histiocytosis associated with massive lymphadenopathy or lymphoproliferative processes, such as Hodgkin's disease and T-cell or B-cell lymphomas. These hematological syndromes can cause the same clinical syndrome as seen in visceral leishmaniasis (VL) $(2,3)$. Our patient with VL did not present with lymphadenopathy, and there had been no family history of such a disorder, making those hematologic processes unlikely. Hemophagocytosis may also occur in serious infections such as brucellosis, tuberculosis, rubella, Epstein-Barr virus, Cytomegalovirus, schistosomiasis, salmonellosis and leishmaniasis $(1,2)$. Brucella species cultures and serology were negative in our patient, as were mycobacterial cultures, polymerase chain reaction for Mycobacterium tuberculosis complex, Salmonella species cultures and various viral serological assays (although the patient's immunocompromised state may have affected the reliability of such serological studies). Pancytopenia can also present in autoimmune diseases, especially systemic lupus erythematosus, but negative antinuclear antibody and anti-DNA tests made this group of illnesses unlikely.

Only very prolonged and thorough review of the bone marrow aspirate produced the correct diagnosis in this patient. The diagnosis of $\mathrm{VL}$ is based on the demonstration of amastigotes in tissue samples (eg, liver, spleen and bone marrow) or the identification of promastigotes in a tissue culture (4). Specific serology is useful in immunocompetent patients, but seroconversion can be delayed or nonexistent in immunocompromised individuals (5). Polyclonal B-cell activation by this parasite produces hypergammaglobulinemia and high titers of antileishmania antibodies, except when hemophagocytosis develops $(5,6)$. The hemophagocytic syndrome seldom occurs in VL but, if present, parasites become very scarce in bone marrow smears for unexplained reasons and, thus, are difficult to detect $(7,8)$. The diagnosis in the presence of hemophagocytosis is elusive and usually delayed, with one study showing delays of up to 134 days (5). In addition, the absence of parasite visualization may lead to an erroneous diagnosis of one of the hematological neoplasms which display hemophagocytosis, resulting in aggressive chemotherapy treatments and/or bonemarrow allografting, as some authors have reported $(1,2,5,6,8,9)$.

Our patient did not present with hepatosplenomegaly, hypergammaglobulinemia or weight loss, which are usually seen in VL. This may have been due to the previous corticosteroid administration. The normalization of all blood parameters and bone marrow histology after therapy with antimony salts supports Leishmania species as the responsible agent for her syndrome (2). The present case highlights the importance of prolonged and in-depth bone marrow review in immunocompromised patients with pancytopenia. Although unusual, VL may be masked by hemophagocytic events mimicking malignant histiocytosis. Clinicians and pathologists must be aware of this possible association between VL and hemophagocytic syndrome as a diagnostic challenge and include VL in the differential diagnosis of hemophagocytosis, especially in patients living in or having previously travelled to endemic areas. 


\section{REFERENCES}

1. Granert C, Elinder G, Ost A, Henter JI. Kala-azar in a one-year-old Swedish child. Diagnosic difficulties because of active hemophagocytosis. Acta Paediatr 1993;82:794-6.

2. Orta C, Ayats R, Portus M, Cadafalch J. Visceral leishmanisis mimicking histiocytic medullary reticulosis. Sangre 1986;31:90-5.

3. Matzner Y, Behar A, Beeri E, Gunders AE, Hershko C. Systemic leishmaniasis mimicking malignant histiocytosis. Cancer 1979;43:398-402.

4. Pearson RD, De Queiroz Sousa A, Jeronimo SMB. Leishmania species: Visceral (Kala-Azar), cutaneous and mucosal leishmaniasis. In: Mandell LA, Dolin R, Bennett JE, eds. Principles and Practice of Infectious Diseases. Philadelphia: Churchill Livingstone, 2000:2831-45.
5. Gagnaire MH, Galambrun C, Stéphan JL. Hemophagocytic syndrome: A misleading complication of visceral leishmaniasis in children: A series of 12 cases. Pediatrics 2000;106:E58.

6. Marom D, Offer I, Tamary H, Jaffe CL, Garty BZ. Hemophagocytic lymphohistiocytosis associated with visceral leishmaniasis. Pediatr Hematol Oncol 2001;18:65-70.

7. Visceral leishmaniasis as an opportunistic infection. Lancet 1986;1:1094.

8. Serrao Neto A, Neves Sousa E, Valente HB, et al. Un cas original de leishmaniose viscérale. Presse Med 1986;15:1286.

9. Nadrid A, Pousse H, Laradi-Chebil S, et al. Infantile visceral leishmaniasis: Difficult diagnosis in cases complicated by hemophagocytosis. Arch Pediatr 1996;3:881-3. 


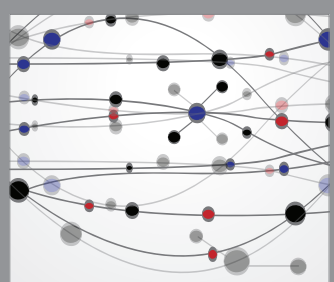

The Scientific World Journal
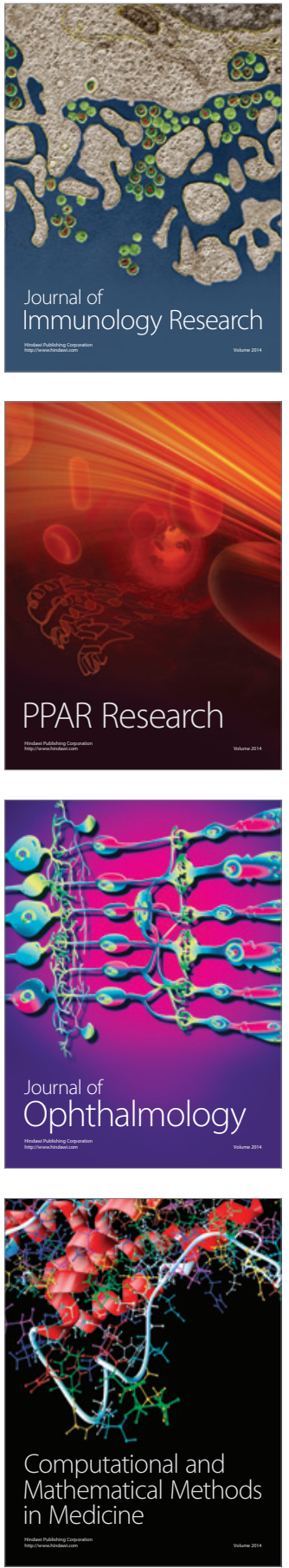

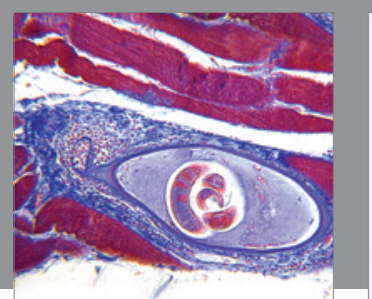

Gastroenterology Research and Practice

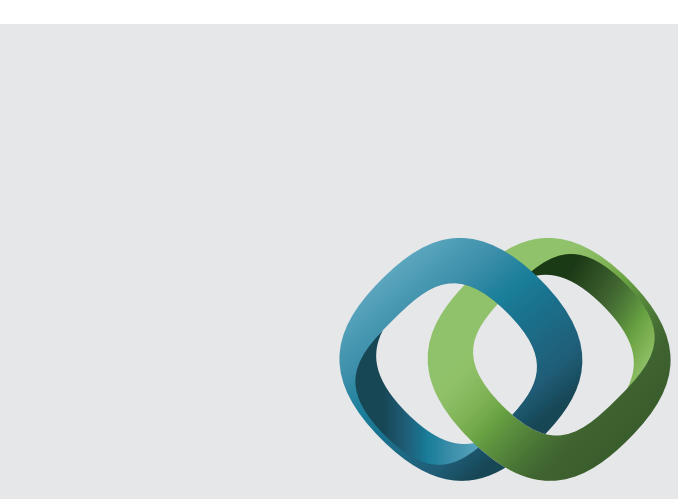

\section{Hindawi}

Submit your manuscripts at

http://www.hindawi.com
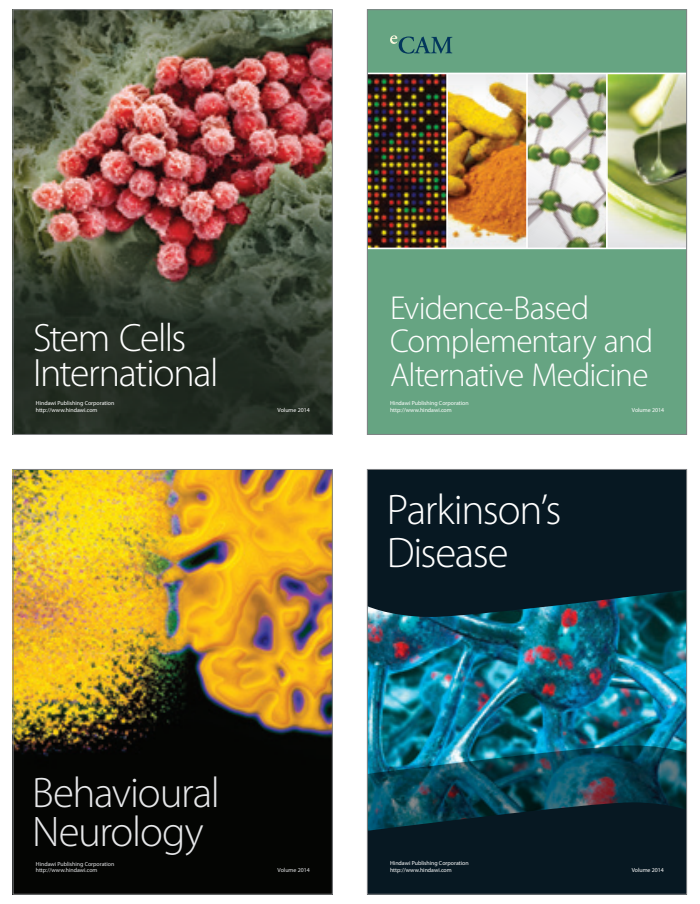
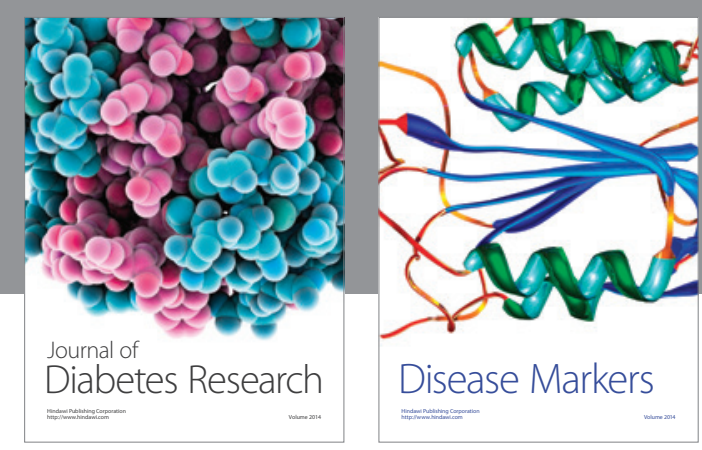

Disease Markers
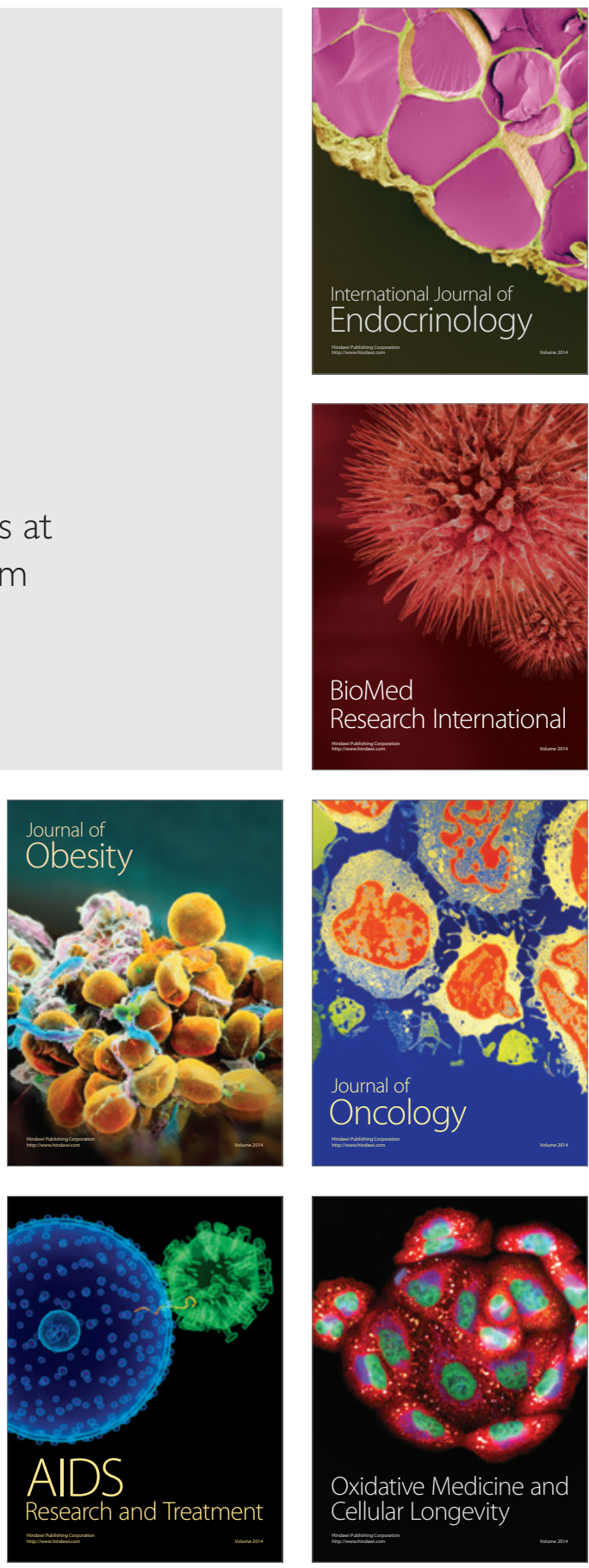